Research Article

\title{
Formulation and Evaluation of Ethosomal Nanoparticles of Avobenzone for Improved Sunscreen Efficacy
}

\author{
Sumit Kumar Nagle*, Mansi Gupta, Deepak Kumar Basedia, B.K.Dubey \\ Technocrats Institute of Technology-Pharmacy, Bhopal, India. \\ *Corresponding author's E-mail: sumitnagle06@gmail.com
}

Received: 05-07-2021; Revised: 24-08-2021; Accepted: 03-09-2021; Published on: 15-09-2021.

\begin{abstract}
Ethosomes entrapping avobenzone were prepared using cold method and the effect of varying concentration of ethanol was considered for obtaining an optimized formulation. Lecithin $(2 \% \mathrm{w} / \mathrm{w})$ was used as the phospholipid to provide the structure to the vesicles and propylene glycol (10\%) was used as the permeating agent. The vesicles were found to be of spherical to irregular shape ranged from $1.11 \mu \mathrm{m}$ to $1.6 \mu \mathrm{m}$ in size. The drug entrapment in the ethosomes was studied by analyzing the unentrapped drug spectrophotometrically. The in vitro permeation study suggested that the maximum permeation in the egg membrane occurred in AET3 $(0.40 \mathrm{mg} / \mathrm{cm} 2)$ with $30 \%$ ethanol concentration. It was observed that only about $2 \%$ degradation occurred at room temperature and all formulations were almost stable at $8^{\circ}$ and $4^{\circ}$ with only $1.3 \%$ degradation of avobenzone thereby proving the stability of the developed system. The best ethosomal formulation (AET3) was incorporated into gel base to obtain sunscreen gels and the results revealed a good protection of the ethosomal gel when $2 \%$ carbopol was used as the gelling base. It could be concluded that incorporation of avobenzone in the ethosomal carrier and formulating the same as gel formulation might help in reducing the dose of avobenzone as well as improving the sunscreen efficacy (sun protection over enhanced duration).
\end{abstract}

Keywords: Ethosome, sunscreen, avobenzone, gel, nanoparticle, lecithin.

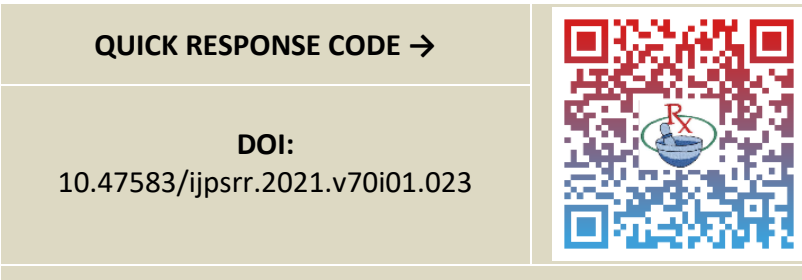

DOI link: http://dx.doi.org/10.47583/ijpsrr.2021.v70i01.023

\section{INTRODUCTION}

E thosomes are lipid vesicles containing phospholipids, alcohol (ethanol and isopropyl alcohol) in relatively high concentration and water. Ethosomes are soft vesicles made of phospholipids and ethanol (in higher quantity) and water. ${ }^{1}$ Ethosomes can entrap drug molecule with various physicochemical characteristics i.e., of hydrophilic, lipophilic, or amphiphilic. The size range of ethosomes may vary from tens of nanometers to microns. ${ }^{1}$ Ethosomes have been widely used for topical, transdermal and transcellular delivery of drugs. ${ }^{2}$ Studies on ethosomal gel have suggested an enhanced duration of action and improved bioavailability of drugs. ${ }^{3-6}$

Avobenzone is a widely used sunscreen molecule incorporated in various sunscreen lotions and creams; but due to the toxicity effects associated with molecule and also the low stability of the oily lotions containing Avobenzone, the shelf life of the formulation decreases. ${ }^{7}$ This leads to decreased sunscreen efficacy of the molecule.
It is widely accepted fact that the nanosized ethosomes have the capacity to improve the stability of incorporated drug molecules. ${ }^{8}$ Hence it was envisioned that incorporating Avobenzone in ethosomes and formulating the sunscreen gels of the same would enable in improved stability and increased sunscreen efficacy and safety of the molecule.

\section{MATERIALS AND METHODS}

Lecithin and Triton X-100 were purchased from HiMedia Laboratories, Mumbai; avobenzone was obtained as gift sample from Gary Pharmaceuticals Pvt. Ltd., Ludhiana. LR/AR/HPLC grade methanol, ethanol and propylene glycol were procured from Oxford Fine Chemicals, Mumbai. All the chemicals were used as received.

\section{Preparation of ethosomes}

Ethosomes were prepared by cold method. In brief the lecithin $(3 \% \mathrm{w} / \mathrm{v})$ was taken in a small round bottom flask and solubilized with ethanol $(10-50 \% \mathrm{v} / \mathrm{v})$ containing drug under mixing with a magnetic stirrer. The round bottom flask was covered to avoid ethanol evaporation. Distilled water was added slowly with continuous stirring to obtain the ethosomal colloidal suspensions. The final suspension of ethosomes was kept at room temperature for $30 \mathrm{~min}$ under continuous stirring. ${ }^{9}$ Formulations were stored in the refrigerator and evaluated for vesicle size, vesicular shape, surface morphology, entrapment efficiency, in vitro drug permeation study and stability study. 
Table 1: Composition of ethosomal formulations

\begin{tabular}{|c|c|c|c|}
\hline Formulation code & Lecithin concentration (\%) & Ethanol concentration (\%) & $\begin{array}{c}\text { Polyethylene glycol } \\
\text { concentration (\%) }\end{array}$ \\
\hline AET1 & 3 & 10 & 10 \\
\hline AET2 & 3 & 20 & 10 \\
\hline AET3 & 3 & 30 & 10 \\
\hline AET4 & 3 & 40 & 10 \\
\hline AET5 & 3 & 50 & 10 \\
\hline
\end{tabular}

\section{Evaluation of ethosomes}

\section{Shape and size}

An optical microscope (Mkow) with a camera attachment was used to observe the shape of the prepared ethosomes formulation. Size and size distribution were determined by dynamic light scattering (DLS) using a computerized inspection system (Malvern Zetasizer).

\section{Entrapment efficiency}

Aliquots of ethosomal dispersion were subjected to centrifugation using cooling ultracentrifuge (Remi) at $12000 \mathrm{rpm}$. The clear supernatant was siphoned off carefully to separate the unentrapped avobenzone and the absorbance was recorded at $\lambda \max 357 \mathrm{~nm}$ using UV/Vis spectrophotometer (Shimadzu UV 1700). Sediment was treated with $1 \mathrm{ml}$ of $0.1 \%$ Triton $X 100$ to lyse the vesicles and then diluted to $100 \mathrm{ml}$ with methanol and absorbance was taken at $357 \mathrm{~nm}$. Amount of avobenzone in supernatant and sediment gave a total amount of avobenzone in $1 \mathrm{ml}$ dispersion. The percent entrapment was calculated using the formula,

$\%$ entrapment $=$ amount of avobenzone in sediment/amount of avobenzone added $\times 100$

\section{In vitro drug permeation study}

The in vitro permeation study was carried out by using modified Franz diffusion cell with egg membrane. The study was performed with phosphate buffer saline $(\mathrm{pH}$ 7.4). The formulation was placed (equivalent to $2.5 \mathrm{mg}$ of drug) on the upper side of skin in donor compartment. The temperature of the assembly was maintained at $37 \pm 2 \circ$. Samples were withdrawn after every hour from the receptor media through the sampling tube and at the same time, same amount of fresh receptor media was added to make sink condition. Withdrawn samples were analyzed for avobenzone constant using UV/Vis spectrophotometer.

\section{Stability study}

Optimized ethosomal formulations were selected for stability study. Formulations were stored at $4 \pm 2^{\circ}, 8^{\circ}$ and at room temperature. Percent drug entrapment was determined at different time intervals (1, 15, 30 and $45 \mathrm{~d})$.

\section{Formulation of ethosomal gel}

Gel formulations were prepared by soaking varying concentration of Carbopol 934 in water for $24 \mathrm{~h}$. The ethosomes equivalent to $2 \% \mathrm{w} / \mathrm{w}$ avobenzone were dissolved in ethanol and was added to the gel with continuous stirring. The plasticizer and other ingredients were added and stirred to obtain the gel noisome loaded gel formulation.

Table 2: Composition of gel formulation

\begin{tabular}{|c|c|c|c|c|}
\hline Ingredients & EG1 & EG2 & EG3 & EG4 \\
\hline Avobenzone ethosome $(\%)$ & 2 & 2 & 2 & 2 \\
\hline Carbopol 934 (\%w/w) & 1 & 2 & 3 & 4 \\
\hline Propylene glycol (\% w/w) & 10 & 10 & 10 & 10 \\
\hline Ethanol (mL) & 5 & 5 & 5 & 5 \\
\hline Triethanolamine $(\% \mathrm{w} / \mathrm{w})$ & 0.7 & 0.7 & 0.7 & 0.7 \\
\hline Water $(\mathrm{g})$ & 15 & 15 & 15 & 15 \\
\hline
\end{tabular}

\section{Evaluation of the ethosomal gel ${ }^{10,11}$}

\section{$\mathrm{pH}$ of the formulation}

Accurately weighed quantity of $5 \mathrm{~g}$ of each gel formulation was mixed separately with $45 \mathrm{~mL}$ of distilled water and the
$\mathrm{pH}$ of the solution was determined with the help of digital $\mathrm{pH}$ meter. 


\section{Viscosity measurement}

The viscosity of each formulation was measured at $10 \mathrm{rpm}$ by using Brookfield DV-1 viscometer employing a S94 spindle.

\section{Spreadability}

Spreadability of the formulations was determined using indigenously developed apparatus. The apparatus consisted of a wooden block provided with a pulley at a one end. A rectangular ground glass was fixed on the block. An excess of cream (3-5 g) was placed on this plate sandwiched using another glass plate having the dimensions as that of fixed ground plate. A $1 \mathrm{~kg}$ weight was placed on the top of the plates for 5 minutes to expel air and to provide a uniform film of the cream between the plates. Excess of the ointment was scrapped off from the edges. Weight of $80 \mathrm{~g}$ was hung on the hook of the top plate with the help of string attached to the hook and the time (in seconds) required by top plate to cover a distance of $10 \mathrm{~cm}$ was noted. Spreadability of the formulation was determined by the following formula:

$\mathrm{S}=\mathrm{M} * \mathrm{~L} / \mathrm{T}$

where,

S- spreadability

$\mathrm{L}$ - distance travelled by the glass slide

$\mathrm{T}$ - time in seconds

$\mathrm{M}$ - weight in the pan

\section{Tube extrudability}

The formulations were filled in clean, lacquered aluminum collapsible tubes with nozzle of $5 \mathrm{~mm}$ opening and pressure was applied on the tubes with the help of finger. Tube extrudability was determined by measuring amount of cream that extruded through the tip when the pressure was applied on tube.

\section{Sunscreen Efficacy Testing ${ }^{12}$}

A solution $0.05 \% \mathrm{w} / \mathrm{w}$ of sodium nitroprusside was prepared in distilled water and $40 \mathrm{ml}$ of this solution was placed in the petriplates. These petriplates were covered with a cellophane membrane. One petriplate containing sodium nitroprusside solution was left uncovered to expose it directly to sunlight. Then $2 \mathrm{~g}$ of the preparation were spread uniformly over the membrane as a layer. The petriplates were exposed to sunlight for $2 \mathrm{~h}$ during midday. After exposure to sunlight, the samples were analyzed using UV method for absorbance of sodium nitroprusside at $395 \mathrm{~nm}$.

\section{RESULTS AND DISCUSSION}

The ethosomes were evaluated for shape and size, entrapment efficiency and in vitro permeation through egg membrane.

\section{Vesicle shape and size}

The vesicles were found to be of spherical to irregular shape ranged from $1.11 \mu \mathrm{m}$ to $1.6 \mu \mathrm{m}$ in size. The smallest particle size was found to be the formulation AET5 whereas the largest size was found to be of AET3.

\section{Entrapment and drug permeation}

The entrapment efficiency of ethosomes was determined for all formulations. Effect of ethanol concentration was observed on percent drug entrapment of ethosomes. The maximum entrapment efficiency was found to be $90.12 \%$ for formulation AET3 and minimum $55.17 \%$ for formulation AET5, respectively. An increase in percent drug entrapment was observed with an increase in ethanol concentration, but when ethanol concentration exceeded $30 \%$, a decrease in percent drug entrapment was observed. Improvement in aqueous solubility of avobenzone was achieved with higher concentration of ethanol, which could be due to its co-solvent effect. Therefore, the more drug amount could be accommodated in the aqueous core of the vesicles however, as the concentration of ethanol increased above $30 \%$ resulting into leakage of drug from fluidized bilayer of vesicles.

Table 3: Evaluation parameters of ethosomes

\begin{tabular}{|c|c|c|c|c|}
\hline Formulation Code & $\begin{array}{l}\text { Vesicle Size } \\
\quad(\mu \mathrm{m})\end{array}$ & Shape & Drug Entrapment (\%) & $\begin{array}{l}\text { Cumulative amount of drug } \\
\text { permeated }\left(\mathrm{mg} / \mathrm{cm}^{2}\right)\end{array}$ \\
\hline AET1 & 1.37 & Irregular & 81.53 & 0.32 \\
\hline AET2 & 1.44 & Spherical & 86.67 & 0.36 \\
\hline AET3 & 1.60 & Spherical & 90.12 & 0.40 \\
\hline AET4 & 1.32 & Irregular & 71.69 & 0.35 \\
\hline AET5 & 1.11 & Irregular & 55.17 & 0.29 \\
\hline
\end{tabular}




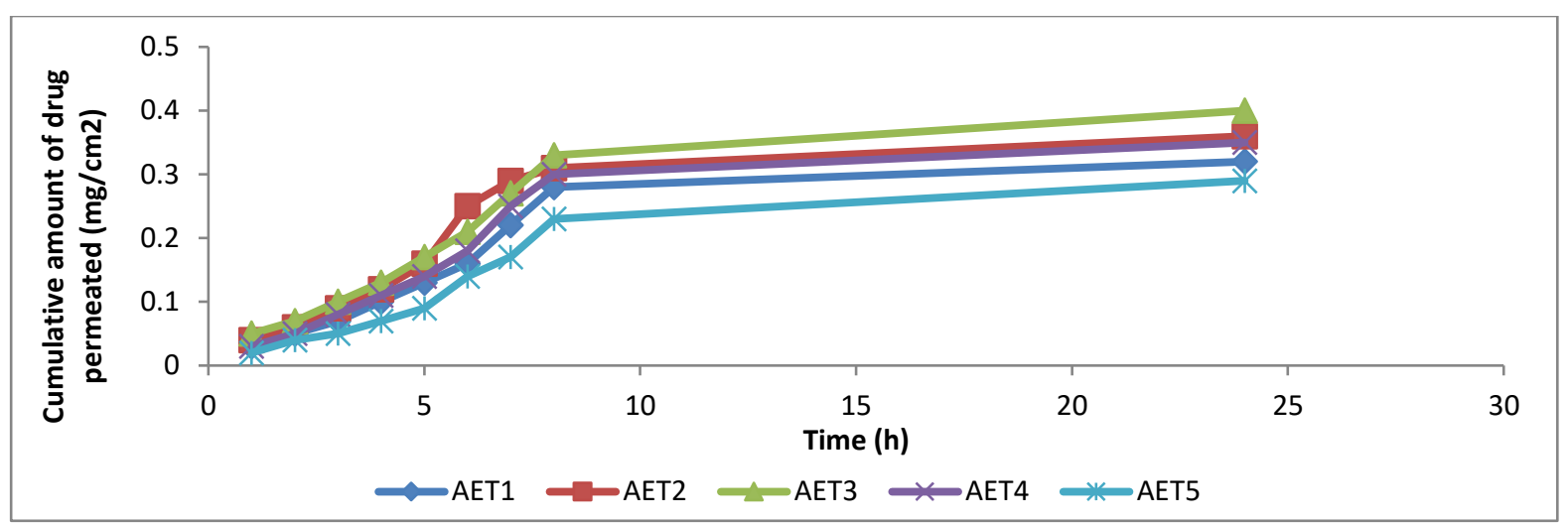

Figure 1: Drug permeation from avobenzone ethosomes

\section{Stability study}

The optimized formulation (AET3) was selected for stability study of vesicles at different temperatures. The formulation was stored in amber glass container at different temperature. The drug content after treatment with triton $\mathrm{X} 100$ and \% residue of avobenzone was calculated as given in Table 4. It was observed that only about $2 \%$ degradation occurred at room temperature and all formulations were almost stable at $8^{\circ}$ and $4^{\circ}$ with only $1.3 \%$ degradation of avobenzone thereby proving the stability of the developed system.

Table 4: Stability of the ethosomes (AET3) on storage

Time (d)
1
15
30
45

$4^{\circ}$
89.23
88.91
88.86
88.76

\section{Drug entrapment (\%)}

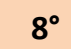

89.86

89.12

88.97

88.89

\section{Room Temperature}

89.17

89.08

88.74

88.31

\section{Evaluation of the ethosomal gel}

The gel formulations were prepared using four concentrations of the gelling agent and were evaluated for physical appearance, $\mathrm{pH}$, viscosity, drug content and in vitro diffusion of the drug.

The gel formulations were found to be off-white in color, homogenous and sticky in feel. The $\mathrm{pH}$ of the all the formulations was between 6.3-6.8, rendering them suitable for topical application. The formulations were found to possess sufficient viscosity to make then suitable for application to the surface and extrusion from the collapsible tube in which they were packed.

The drug content in all the formulations ranged from 96.7 to $99.21 \%$ confirming the incorporation of the ethosomes into the gel base. The results of the evaluation parameters are presented in Table 5.

Table 5: Evaluation of the gel formulations

\begin{tabular}{|c|l|l|l|c|c|c|c|}
\hline $\begin{array}{c}\text { Formulation } \\
\text { code }\end{array}$ & Colour & Appearance & $\mathbf{p H}$ & $\begin{array}{c}\text { Viscosity } \\
\text { (cps) }\end{array}$ & $\begin{array}{c}\text { Drug content } \\
\text { (\%) }\end{array}$ & $\begin{array}{c}\text { Spreadability } \\
\text { (g.cm/sec) }\end{array}$ & $\begin{array}{c}\text { Extrudability } \\
\text { (\%) }\end{array}$ \\
\hline EG1 & Off White & Sticky & 6.34 & 7986 & 96.7 & 17.12 & 95 \\
\hline EG2 & Off White & Sticky & 6.76 & 8270 & 99.21 & 16.08 & 84 \\
\hline EG3 & Off White & Sticky & 6.47 & 8148 & 97.51 & 18.65 & 92 \\
\hline EG4 & Off White & Sticky & 6.54 & 8612 & 98.79 & 15.26 & 87 \\
\hline
\end{tabular}

\section{Sunscreen efficacy}

The sunscreen efficacy of the ethosomal gel formulations were evaluated using sodium nitroprusside method and the results is reported in Table 6.

Sun exposure method using sodium nitroprusside solution was used for testing the in vitro sunscreen efficacy of the formulations. Sodium nitroprusside is known to be photosensitive in aqueous solution and on exposure to direct sunlight it degrades to yield prussian blue and nitric oxide (NO). The spectrophotometric measurement has been employed to determine the stability of sodium nitroprusside; with most emphasis on increase in the absorbance at 390-395 $\mathrm{nm}$ with degradation. It was observed that formulation EG2 had the best sunscreen efficacy. 
Table 6: Sunscreen efficacy measured as absorbance of sodium nitroprusside solution at $395 \mathrm{~nm}$.

\begin{tabular}{|c|c|}
\hline Formulation & $\begin{array}{c}\text { Absorbance of sodium } \\
\text { nitroprusside solution }\end{array}$ \\
\hline EG1 & 2.684 \\
\hline EG2 & 1.002 \\
\hline EG3 & 1.662 \\
\hline EG4 & 2.026 \\
\hline $\begin{array}{c}\text { Covered with } \\
\text { cellophane membrane } \\
\text { only }\end{array}$ \\
\hline
\end{tabular}

\section{CONCLUSION}

The use of ethosomal flexible carriers has gained popularity as promising approach for transdermal drug delivery. Incorporation of avobenzone in the ethosomal carrier and formulating the same as gel formulation might help in reducing the dose of avobenzone as well as improving the sunscreen efficacy (sun protection over enhanced duration).

\section{REFERENCES}

1. Bhalaria MK, Naik S, Misra AN. Ethosomes: A novel delivery system for antifungal drugs in the treatment of topical fungal diseases. Indian J Exper Biol. 2009; 47: 368-375.

2. Kumar KP, Radhika PR, Sivakumar T. Ethosomes-A Priority in Transdermal Drug Delivery. Int J Advances Pharm Sci. 2010; 1: 111-121.

3. Barupal AK, Gupta V, Ramteke S. Preparation and Characterization of Ethosomes for Topical delivery of Aceclofenac. Indian J Pharm Sci. 2010; 72(5): 582-86
4. El-Shenawy AA, Abdelhafez WA, Ismail A, Kassem AA. Formulation and Characterization of Nanosized Ethosomal Formulations of Antigout Model Drug (Febuxostat) Prepared by Cold Method: In Vitro/Ex Vivo and In Vivo Assessment. AAPS PharmSciTech. 2020; 21: 31-43

5. Limsuwan $T$, Boonme $P$, Khongkow $P$, Amnuaikit $T$. Ethosomes of Phenylethyl Resorcinol as Vesicular Delivery System for Skin Lightening Applications. BioMed Res Int. 2017; doi.org/10.1155/2017/8310979

6. Mohammed MI, Makky AMA, Abdellatif MM. Formulation and characterization of ethosomes bearing vancomycin hydrochloride for transdermal delivery. Int J Pharm Pharm Sci. 2014; 6(11): 190-4

7. Gasparro FP. Sunscreens, skin photobiology, and skin cancer: the need for UVA protection and evaluation of efficacy. Environ Health Perspect. 2000; 108 Suppl. 1: 718.

8. Akiladei D, Basak S. Ethosomes a noninvasive approach for transdermal drug delivery. Int J Curr Pharm Res. 2010; 2: 14.

9. Mustofa RR, Iskandarsyah. Preparation and characterization of anti-acne ethosomes using cold and thin-layer hydration methods. Int J Appl Pharmaceut. 2018; 10(S1): 339-342

10. Panda $P$, Ghosh A. Formulation and Evaluation of Topical Dosage Form of Eupatorium Odoratum Linn and Their Wound Healing Activity. Int J Pharm BioSci. 2010; 1(2): 201203.

11. Pandey T, Kumar A, Sharma R. Effect of essential oils as penetration enhancers for ibuprofen loaded transdermal gel formulations. J Pharmacol Biomed. 2021; 5(2): 268-276

12. Kaidbey $\mathrm{KH}, \mathrm{KligmanAM}$. Laboratory methods for appraising the efficacy of sunscreens. J SocCosmet Chem. 1978; 29: 525-536.

Source of Support: The author(s) received no financial support for the research, authorship, and/or publication of this article.

Conflict of Interest: The author(s) declared no potential conflicts of interest with respect to the research, authorship, and/or publication of this article.

For any question relates to this article, please reach us at: editor@globalresearchonline.net New manuscripts for publication can be submitted at: submit@globalresearchonline.net and submit_ijpsrr@rediffmail.com 